\title{
An adaptive psychophysical method for subject classification
}

\author{
ALAN B. COBO-LEWIS \\ University of Miami, Coral Gables, Florida
}

\begin{abstract}
In psychophysical experiments, one's goal is usually to measure some continuous parameter hypothesized to determine the statistical properties of a subject's responses. Methods are well developed that adaptively manipulate stimulus characteristics in such a way that the reliability of the parameter estimate is maximized. However, such methods are inapplicable in situations in which the goal is to assign subjects to discrete categories, rather than to measure a continuous parameter. This paper introduces a technique that is directly applicable to efficient categorization and that adaptively manipulates stimulus characteristics in such a way that the information obtained from each trial is maximized. This technique is based on the principle of minimum estimated expected entropy, whereby stimulus parameters on each trial are chosen in order to minimize the estimated expected entropy of the a posteriori probability distribution that expresses how likely a subject is to belong to each of a group of mutually exclusive categories. A sample implementation of the technique-the classification of infant subjects according to their audiograms-is then described and evaluated via computer simulation.
\end{abstract}

In psychophysical experiments, one's goal is usually to measure some continuous subject parameter hypothesized to determine the statistical properties of that subject's responses to a given class of stimuli. For example, one might be interested in determining the threshold intensity such that the subject is likely to detect stimuli with intensities above that threshold but is unlikely to detect stimuli with intensities below that threshold. In these types of experiments, one generally wants the estimate of the subject parameter to be as reliable as possible. Two approaches can be taken to achieve this goal. The first approach is to estimate the parameter of interest from all of the available data - that is, from the subject's responses to all the stimuli presented. One can accomplish this by, for example, least squares fitting or maximum-likelihood (ML) fitting. The second approach is to choose the stimulus parameters in such a way that the subject's responses are likely to be maximally informative. Although the two approaches are logically independent, many psychophysical methods take both approaches simultaneously.

Psychophysical methods are particularly well developed for experiments in which threshold is the subject parameter of interest. In these experiments, test stimuli close to threshold are generally more informative than those that are far from threshold (Taylor, 1971). However,

This work was supported by NIH/NICHD Grant R43 HD30623 to Intelligent Hearing Systems, Miami, FL (E. Miskiel, principal investigator), and by NSF Grant 9617767 (A.B.C.-L., principal investigator). Thanks to Rafael Delgado, Rebecca Eilers, Andrew B. Watson, Bernhard Treutwein, and an anonymous reviewer for comments on earlier versions of this manuscript, and to Carlos Lopez, Craig Mason, Kim Oller, Özcan Özdamar, and Michele Steffens for helpful comments on this topic. Correspondence should be addressed to A. Cobo-Lewis, Psychology Annex. University of Miami, PO Box 249229. Coral Gables, FL 33124-0721 (e-mail; acobolew@peds.med.miami.edu). a subject's threshold is unknown at the start of an experiment. (Otherwise, of course, there would be no need to conduct the experiment.) Thus, in threshold experiments, one must solve the bootstrap problem of estimating threshold in order to place stimuli near that threshold so that it can be estimated. One solution is to implement an adaptive method, which selects stimulus parameters on the basis of the history of the subject's responses to preceding stimuli. Because adaptive methods are designed to present most stimuli near threshold, they are typically more efficient than nonadaptive methods (Watson \& Fitzhugh, 1990).

There are many adaptive methods (for a review, see Treutwein, 1995). Perhaps the most basic is the simple staircase (Cornsweet, 1962), whereby the stimulus parameter is increased by one step following a correct subject response and is decreased by one step following an incorrect subject response. Modified staircases may feature waiting for multiple correct or incorrect responses before the stimulus parameter is adjusted (Levitt, 1971; Wetherill \& Levitt, 1965) or reducing step size systematically as the experiment progresses (Johnson \& Shapiro, 1989; Robbins \& Monro, 1951; Tyrrell \& Owens, 1988). Or, selection of the stimulus parameter may be based on the results from blocks of trials (Findlay, 1978; Hall, 1981; Taylor \& Creelman, 1967; Watt \& Andrews, 1981).

Thus far, we have considered estimating the subject parameter of interest from all of the available data as a separate approach from choosing stimulus parameters to be maximally informative. For threshold experiments, a particularly efficient approach to achieving the overarching goal of maximizing the reliability of the subject parameter's estimate is to use both approaches simultaneously. This is the approach taken by adaptive ML methods (Green, 1993; Hall, 1968; Pentland, 1980; Watson \& Pelli, 
1983), in which the current most likely value of threshold is determined after each trial (thus taking the first approach), and this value is selected as the next value for the stimulus parameter (thus taking the second approach). Perhaps the most widely used adaptive ML method is QUEST (Watson \& Pelli, 1983).

The ultimate goal of these procedures is to maximize the reliability of the estimate of a subject parameter, but adaptive ML procedures do not necessarily maximize reliability. The minimum-variance method (MVM; KingSmith, 1984) is a modification of QUEST (Watson \& Pelli, 1983) whereby instead of setting the stimulus parameter equal to the maximally likely value of the subject parameter, one sets the stimulus parameter equal to whatever value minimizes the subject parameter's estimated expected variance at the end of the next trial. Thus, MVM addresses the problem of reliability directly. Indeed, it addresses the problem successfully; numerical simulations have revealed that it is more efficient than several versions of QUEST (King-Smith, 1984; King-Smith, Grigsby, Vingrys, Benes, \& Supowit, 1994). An extension of MVM is the ideal psychometric procedure (IPP; Pelli, 1987), whereby one adaptively sets the stimulus parameter equal to whatever value minimizes the subject parameter's estimated expected variance at the end of the last trial. However, a practical drawback to IPP is that the required computation time is exponential in the number of trials.

What if the subject parameter of interest is not threshold? For example, what if one wishes to measure the slope of a subject's psychometric function or the baseline probability of a false alarm? In neither of these two examples does the subject parameter carry the same units as the stimulus parameter. Although ML methods such as QUEST do enable one to calculate estimates of such subject parameters, they offer little guidance on how to select stimulus parameters in such a way that the estimates of these nonthreshold subject parameters be maximally reliable. This is because adaptive ML methods endeavor to set the stimulus parameter (e.g., a stimulus intensity) equal to a likely value of the subject parameter (e.g., a threshold intensity). But, the subject parameter and the stimulus parameter may be incommensurate; for example, they may carry different units (to repeat the two examples that began this paragraph, a psychometric function's slope might carry units of 1 intensity, and a baseline false-alarm probability would be dimensionless). In such cases, adaptive ML methods such as QUEST cannot set the stimulus parameter equal to the subject parameter. Because MVM and IPP do not rely on setting the stimulus parameter equal to a likely value of the subject parameter, MVM and IPP are not hampered by this limitation. Thus, MVM and IPP have an additional advantage: The subject parameter of which a reliable estimate is desired need not be of the same type as the stimulus parameter available for adaptive adjustment. In fact, with these two procedures, multiple stimulus parameters can be available for adaptive adjustment, and none of them need be of the same type as the subject parameter.
Although MVM and IPP relax the restriction that the stimulus parameter under adaptive adjustment be of the same type as the subject parameter whose reliability is being maximized, they are still applicable only to continuous subject parameters. All the aforementioned methods are inapplicable when the subject parameter of interest is categorical-that is, when it possesses only nominal properties (Stevens, 1951). Even MVM and IPP, which have the broadest applicability, are inapplicable here because when the subject parameter merely represents membership in a discrete category, its variance is undefined.

This paper introduces a technique that is directly applicable to efficient categorization and that adaptively selects stimulus parameters in such a way that the information obtained from each trial is maximized. This technique is based on the principle of minimum estimated expected entropy (MEEE), whereby stimulus parameters on each trial are chosen to minimize the estimated expected entropy of the a posteriori probability distribution across categories. The technique is described, and then a sample implementation of the technique-categorization of infant audiograms - is presented and evaluated via computer simulation.

\section{THEORY}

\section{Dynamic Update of a Posteriori Probabilities}

Assume that the experimenter's goal is to classify the subject into one of a number of categories. On the basis of (1) the respective estimated a priori probabilities that a subject belongs to the various categories and (2) the measured responses of the subject, we follow the adaptive Bayesian approach of updating our estimates of the respective a posteriori probabilities for each category. If we assume trial-by-trial response independence, then after trial $t$, for any given category $c$, the conditional probability of obtaining a particular set of responses across the $t$ trials is

$$
\operatorname{Pr}(\text { obtained data } \mid \mathbf{C}=c)=\prod_{\tau=1}^{t} \operatorname{Pr}\left(\mathbf{R}_{\tau}=R_{\tau} \mid \mathbf{C}=c\right),
$$

where $\operatorname{Pr}\left(\mathbf{R}_{\tau}=R_{\tau} \mid \mathbf{C}=c\right)$ represents the conditional probability that $\mathbf{R}_{\tau}$, the response to trial $\tau$, equals $R_{\tau}$, the obtained response on trial $\tau$, given that the correct category, $\mathbf{C}$, is category $c$. (Here and following, random variables are bold-faced.) Trial-by-trial response independence is a common assumption that permits expression of the likelihood of the entire data set as the product of the likelihoods of the individual trials.

Our immediate goal is to derive an expression for the probability that $c$ is the correct category, given the obtained data. What Equation 1 provides is the obverse: the probability of the obtained data, given that $c$ is the correct category. To calculate the desired probability after trial $t$, we apply Bayes's theorem, thus expressing $p_{c}^{(t)}$, the desired a posteriori probability for category $c$, as 


$$
p_{c}^{(t)}=\frac{p_{c}^{(0)} \prod_{\tau=1}^{t} \operatorname{Pr}\left(\mathbf{R}_{\tau}=R_{\tau} \mid \mathbf{C}=c\right)}{\sum_{\gamma}\left[p_{\gamma}^{(0)} \prod_{\tau=1}^{t} \operatorname{Pr}\left(\mathbf{R}_{\tau}=R_{\tau} \mid \mathbf{C}=\gamma\right)\right]},
$$

where $p_{c}^{(0)}$ represents the a priori probability (probability immediately after trial 0 , i.e., before testing has commenced) that $c$ is the correct category. (Superscript indices are in parentheses in order to distinguish them from exponents.) Equation 2 simply represents the direct application of Bayes's theorem: $\operatorname{Pr}\left(A_{i} \mid B\right)=\operatorname{Pr}\left(B \mid A_{i}\right) \operatorname{Pr}\left(A_{i}\right) /$ $\sum_{j} \operatorname{Pr}\left(B \mid A_{j}\right) \operatorname{Pr}\left(A_{j}\right)$. Although a posteriori weights are more familiarly expressed in the Bayesian form of Equation 2, it is more convenient computationally to express them via the recurrence relation

$$
p_{c}^{(t)}=\frac{p_{c}^{(t-1)} \operatorname{Pr}\left(\mathbf{R}_{t}=R_{t} \mid \mathbf{C}=c\right)}{\sum_{\gamma}\left[p_{\gamma}^{(t-1)} \operatorname{Pr}\left(\mathbf{R}_{t}=R_{t} \mid \mathbf{C}=\gamma\right)\right]},
$$

which is derived in Appendix A.

The distribution of the random variable $\mathbf{R}_{t}$, which represents the subject's response to a given trial, is simply the psychometric function. The psychometric function depends on the subject's category, $\mathbf{C}$, and on the stimulus parameters for the given trial. It is convenient to collapse the stimulus parameters subject to manipulation into the single $\mathrm{N}$-dimensional stimulus parameter $s$. Category $c$ 's psychometric function, then, is written simply as $\psi_{c}(s)$. For simplicity, we consider only binary responses (e.g., go/no-go), although the method is easily extended to $n$-ary responses. We will term one response success and the other response failure.

\section{Stimulus Selection}

We wish to maximize the reliability of our measurement. Recall that, if our subject parameter were numerical (i.e., if it possessed at least interval properties), we might accomplish this by using MVM to drive the subject parameter's estimated variance ever lower. But, in the current problem, our subject parameter is instead categorical (i.e., it possesses only nominal properties). Variance therefore becomes an inapplicable measure, and our information about the subject parameter's value is summarized by a probability distribution across categories (the $p_{c}^{(t)} \mathrm{s}$ ). How to summarize uncertainty across such a nominal scale? One applicable measure is entropy. Specifically, one can quantify the uncertainty about the correct category immediately before trial $t$ (immediately after trial $t-1)$ as the entropy of the distribution of the dynamic a posteriori probabilities,

$$
H^{(t-1)}=-\sum_{c} p_{c}^{(t-1)} \log p_{c}^{(t-1)} .
$$

(The base of the logarithm in Equation 4 is arbitrary. It is typically chosen to be 2 , in which case the entropy has units of bits.) Intuitively, Equation 4 can be viewed as the expected value, across categories, of $-\log p_{c}^{(t-1)}$, which, in turn, expresses how "surprised" we should be to discover that the subject is actually a member of category $c$. If the probability of category $c$ is unity (i.e., if there is utter certainty that the subject belongs to category $c$ ), then $-\log p_{c}^{(t-1)}$ evaluates to $-\log 1=0$, for membership in a certain category should be completely unsurprising. On the other hand, if the probability of category $c$ is zero (i.e., if there is no chance that the subject belongs to category $c$ ), then $-\log p_{c}^{(t-1)}$ evaluates to $-\log 0=\infty$, for membership in an impossible category should be infinitely surprising. If the probability of category $c$ lies somewhere between 0 and 1 , then $-\log p_{c}^{(t-1)}$ evaluates to some finite positive number that quantifies how surprised we should be to discover that a subject is actually a member of category $c$.

If there is utter certainty about a subject's category membership, the entropy (Equation 4) evaluates to zero. [Verification of this result requires knowledge of the mathematical identity $\lim _{p \rightarrow 0}(p \log p)=0$.] If, on the other hand, there is complete uncertainty about a subject's category membership (i.e., if the distribution across categories is uniform, so that $p_{c}^{(t-1)}$ is constant across $c \mathrm{~s}$ ), then entropy evaluates to its maximal value, which is $\log c$. Intermediate certainty yields intermediate entropy: The lower the entropy, the more information there is about the subject's category. Thus, for our adaptive method, the natural goal is to present stimuli that tend to drive the entropy ever lower, for our goal is to minimize our uncertainty about the subject's category.

We compute two sets of conditional a posteriori probabilities before every trial $t$ : Category $c$ 's probability, contingent on a success in response to stimulus $s$ on trial $t$, is written as $\breve{p}_{c}^{(t)}(s)$; category $c$ 's probability, contingent on failure, is written as $\breve{p}_{c}^{(t)}(s)$. (The diacritics function as mnemonic aids; the diacritic for success should be pronounced "smile," and the diacritic for failure should be pronounced "frown.") These conditional probabilities are then used to define conditional entropies. The conditional entropy after trial $t$, contingent on a success response to stimulus $s$, is defined as

$$
\bar{H}^{(t)}(s)=-\sum_{c} \breve{p}_{c}^{(t)}(s) \log \bar{p}_{c}^{(t)}(s),
$$

and the conditional entropy after trial $t$, contingent on a failure response to stimulus $s$, is defined as

$$
\hat{H}^{(t)}(s)=-\sum_{c} \hat{p}_{c}^{(t)}(s) \log \bar{p}_{c}^{(t)}(s) .
$$

After trial $t-1$, the subject's success probability in response to stimulus $s$ can be estimated as the weighted mean of the psychometric functions for all the categories:

$$
\bar{\psi}^{(t-1)}(s)=\sum_{c} p_{c}^{(t-1)} \psi_{c}(s),
$$

whose weights are given by the current estimates of the categories' respective probabilities. These estimated success probabilities are then combined with the conditional entropies of Equations 5 and 6 in order to estimate the ex- 
pected entropy following the subsequent trial, $t$, as a function of the choice for the stimulus parameters, $s$. This estimated expected entropy is defined as

$$
\tilde{H}^{(t)}(s)=\bar{\psi}^{(t-1)}(s) \vec{H}^{(t)}(s)+\left[1-\bar{\psi}^{(t-1)}(s)\right] \bar{H}^{(t)}(s) .
$$

It simply represents the current estimate of the expected entropy after the next trial, as a function of the stimulus to be presented. For computational convenience, it can be re-expressed as

$$
\begin{aligned}
& \tilde{H}^{(t)}(s)= \\
& \quad-\sum_{c} p_{c}^{(t-1)} \log \left[p_{c}^{(t-1)}\left(\frac{1-\psi_{c}(s)}{1-\bar{\psi}^{(t-1)}(s)}\right)^{l-\psi_{c}(s)}\left(\frac{\psi_{c}(s)}{\bar{\psi}^{(t-1)}(s)}\right)^{\psi_{c}(s)}\right],
\end{aligned}
$$

which is derived in Appendix B. (The tilde diacritic is intended to represent a concatenation of the frown and smile diacritics.) Finally, the MEEE principle states that, for each trial $t$, the stimulus parameters, $s$, should be chosen so that the expected entropy, $\tilde{H}^{(t)}(s)$, is minimized. (Barring the analytic derivation of stimulus-parameter values that satisfy this condition, the minimization can be accomplished numerically.) This constitutes the method of minimum estimated expected entropy.

For a $T$-trial experiment, the ideal strategy (IMEEE) would be the analogue of IPP: to choose $s$ so that $\tilde{H}^{(T)}(s)$ is minimized. However, due to the enormous computational cost of IMEEE, we restrict further attention to the simpler MEEE method.

\section{SAMPLE IMPLEMENTATION}

\section{CAST Description}

General approach. In order to allow fast and accurate estimation of audiograms in infants, Özdamar, Eilers, and colleagues developed an algorithm known as CAST (classification of audiograms by sequential testing; Eilers, Özdamar, \& Steffens, 1993; Özdamar, Eilers, Miskiel, \& Widen, 1990). CAST is an automated form of visual reinforcement audiometry (VRA; Liden \& Kankkunen, 1969; Moore, Wilson, \& Thompson, 1977; Suzuki \& Ogiba, 1961) whereby infant subjects are taught to respond to audible stimuli via the use of visual reinforcers. The infant is seated with head facing straight ahead, and sounds are presented via speakers positioned off of midline. An infant who turns his/her head toward the sound is rewarded by the brief illumination and activation of fixed but animated toys (e.g., a stuffed animal sitting at a drum set) that otherwise remain hidden behind smoked Plexiglas.

Standard VRA usually generates too few trials for adequate estimation of an audiogram. However, by careful selection of stimulus parameters, efficient pooling of information across all trials, and application of a priori knowledge about a population's hearing characteristics, CAST seeks to classify an infant into one of nine categories, defined by the prototypical audiograms displayed in Figure 1. Thus, whereas standard VRA is too coarse a technique for audiogram estimation, CAST is sophisticated enough to perform audiogram classification. The efficiency and reliability of CAST have been evaluated by computer simulation (Özdamar et al., 1990) and subsequently confirmed by clinical trial (Eilers et al., 1993).

Specifics of original CAST. Özdamar et al.'s (1990) CAST (henceforth termed original CAST) uses Equation 2 or 3 to dynamically update the a posteriori probability distribution across a subject's possible categories; however, its procedure for adjusting stimulus parameters, although clever, is somewhat ad hoc.

Immediately before each trial $t$, original CAST computes the mean and variance, weighted by the a posteriori probabilities of the the just-completed trial $t-1$, of the threshold estimates for each frequency $f$ as

$$
m^{(t-1)}(f)=\sum_{c} p_{c}^{(t-1)} T_{c, f}
$$

and

$$
v^{(t-1)}(f)=\sum_{c} p_{c}^{(t-1)}\left[T_{c, f}-m^{(t-1)}(f)\right]^{2},
$$

respectively, where $T_{c, f}$ is a constant representing the assumed threshold for audiogram prototype $c$ at frequency $f$. (These are the thresholds plotted in Figure 1.) Original CAST then selects the next trial's stimulus frequency, $f^{\prime}$, as the one with the greatest threshold variance, $v^{(t-1)}\left(f^{\prime}\right)$, and selects the next trial's intensity as the mean threshold, $m^{(t-1)}\left(f^{\prime}\right)$, for that frequency.

Applying the MEEE method to CAST. In the language of the MEEE method, each of CAST's audiogram prototypes represents a subject category. Because CAST adjusts frequency and intensity from trial to trial, the stimulus parameter takes the form of an ordered pair, $s=$ $(f, I)$. It now remains to define the form of the psychometric function. For purposes of analytic convenience, this paper implemented CAST to assume a logistic form,

$$
\psi_{c}(f, I)=\frac{\varpi-\varphi}{1+\exp \left[-k\left(I-T_{c, f}\right)\right]}+\varphi,
$$

where intensities $\left(I\right.$ and $\left.T_{c, f}\right)$ are expressed in decibels, $T_{c, f}$ represents category $c$ 's threshold at frequency $f$ (see Figure 1), $\Phi$ (a variant of a lowercase pi) represents the assumed probability that the subject will succeed in response to an arbitrarily intense stimulus (previous work fixed $\varpi=1), \varphi$ represents the assumed false-alarm rate (previous work fixed $\varphi=0$ ), and the scale parameter was set at $k=0.2 \mathrm{~dB}^{-1}$. With this scale parameter, the logistic function rises from about $5 \%$ of the $\varpi-\varphi$ span to about $95 \%$ of the $\varpi-\varphi$ span, over a range of $30 \mathrm{~dB}$. However, logistic functions may provide poorer fits than do cumulative Gaussians to yes-no detection data $(\mathrm{Gu} \&$ Green, 1994). Consequently, although CAST was implemented to assume a logistic form for the psychometric function, the numerical simulations described below were carried out on the assumption that the subjects' true psychometric function, $\Psi(f, I)$, was actually Gaussian: 


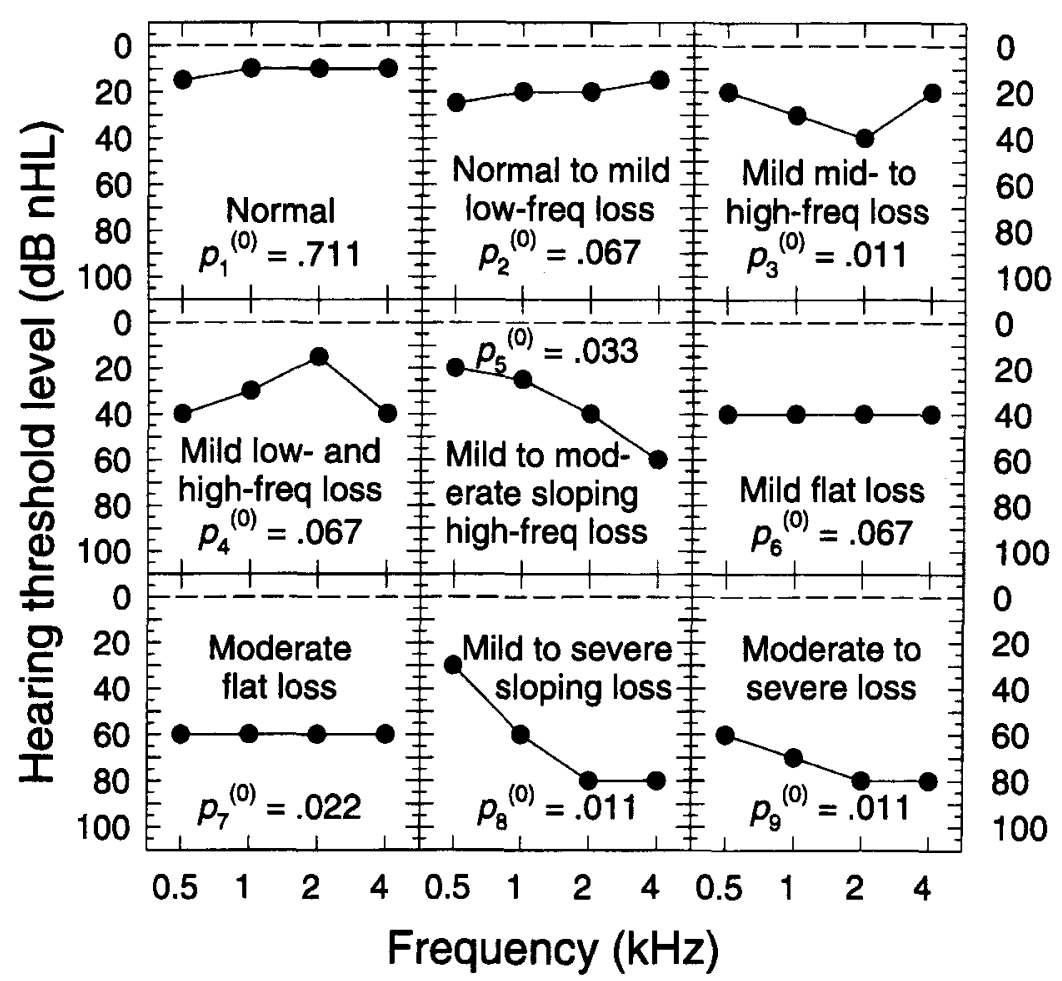

Figure 1. Audiogram prototypes and their associated a priori probabilities $\left(p_{c}^{(0)} \mathbf{s}\right)$. Data from Eilers, Özdamar, and Steffens (1993).

$$
\Psi(f, I)=\frac{\Pi-\Phi}{\sigma \sqrt{2 \pi}} \int_{-\infty}^{l} \exp \left[\frac{\left(i-T_{f}\right)^{2}}{\sigma^{2}}\right] \mathrm{d} i+\Phi,
$$

where $T_{f}$ is the subject's true threshold at frequency $f, \Pi$ represents the probability that the subject will succeed in response to an arbitrarily intense stimulus, and $\Phi$ represents the false-alarm rate. The spread factor of the Gaussian function was chosen to be $\sigma=4 k^{-1}(2 \pi)^{-1 / 2}$, so that the slope at the inflection point would be identical to that of the logistic approximation to the Gaussian function. With this spread factor, the Gaussian function rises from about $3 \%$ of the $\Pi-\Phi$ span to about $97 \%$ of the $\Pi-\Phi$ span, over a range of $30 \mathrm{~dB}$.

The assumed and "true" psychometric functions of Equations 12 and 13 saturate at success-probability $\sigma$ and $\Pi$, respectively. These parameters represent the probability of task orientation (PTO; Eilers, Miskiel, Widen, \& Lopez, 1989; Özdamar et al., 1990), which reflects such factors as subject attention, state, and responsiveness. Many readers will recognize the complement of PTO $(1-\varpi$ and $1-\Pi$, for assumed and true values, respectively) as the false-negative rate, also termed the "lapsing" probability. The PTO represents the basic uncertainty of responses when the subject is under incomplete stimulus control. Different subjects can exhibit different
PTO values, and the PTO value that best describes the behavior of a subject is generally unknown at the outset of testing. Consequently, CAST must make some assumptions regarding PTO. (By fixing $\sigma=1.0$, previous work assumed perfect attention.) The same argument applies to the false-alarm rate $(\varphi$ and $\Phi$, for assumed and true values, respectively). Thus, in addition to making assumptions about PTO, CAST must also make some assumptions about the false-alarm rate. In the investigations described later in this paper, the true PTO, П, was subjected to experimental manipulation between 1.0 , which represents a perfectly attentive subject, and 0.7 , which represents a very inattentive subject (Özdamar et al., 1990 ). The assumed PTO, $\Phi$, was set at 0.85 , representing a moderately inattentive subject, because numerical investigations not detailed here revealed that this was effective at preventing miscategorizations for very inattentive pseudosubjects while not costing a proportionate amount for perfectly attentive pseudosubjects. Similarly, the true false-alarm rate, $\Phi$, was subjected to experimental manipulation between 0.0 and 0.3 , and the assumed false-alarm rate, $\varphi$, was set at 0.15 .

The MEEE method requires that before every trial, the stimulus parameters, $s$, be selected so that the estimated expected entropy, $\tilde{H}$, is minimized. (Appendix C provides details on the numerical methods by which this mini- 
mization was accomplished.) We will use the term MEEECAST to refer to the method whereby CAST is implemented with the modification that the original stimulusselection procedure (Özdamar et al., 1990) is replaced by the MEEE procedure.

A final consideration is that CAST can reach a given stopping criterion before having tested all four frequencies (Özdamar et al., 1990). Consequently, the criteria for choosing stimulus parameters were modified so that, on Trials 2-4, a frequency was excluded from consideration if it had been tested previously. Thus, in the first four trials, each frequency was tested exactly once.

\section{How Original CAST Can Select}

\section{Suboptimal Stimulus Parameters}

It is instructive for understanding both original CAST and MEEE-CAST to consider some of the ways in which original CAST can select suboptimal stimulus parameters.
In this discussion, we simplify matters by (1) neglecting frequency selection, so that we consider only the selection of stimulus intensity, and (2) considering only two categories (i.e., two possible psychometric functions). This simplified situation is diagrammed in Figure 2. In each panel, the psychometric function of the lower threshold category is diagrammed toward the left (toward low intensities), and the psychometric function of the higher threshold category is diagrammed toward the right (toward high intensities). Also plotted is the estimated expected entropy as a function of candidate stimulus intensity, $\tilde{H}(s)$, as calculated from Equation 9. The lower the estimated expected entropy for a candidate intensity, the more informative a trial at that intensity is likely to be. Therefore, MEEE-CAST will test at the intensity where this entropy is minimized, as indicated by the dotted arrowheaded lines. On the other hand, original CAST will test at an intensity representing the weighted average of

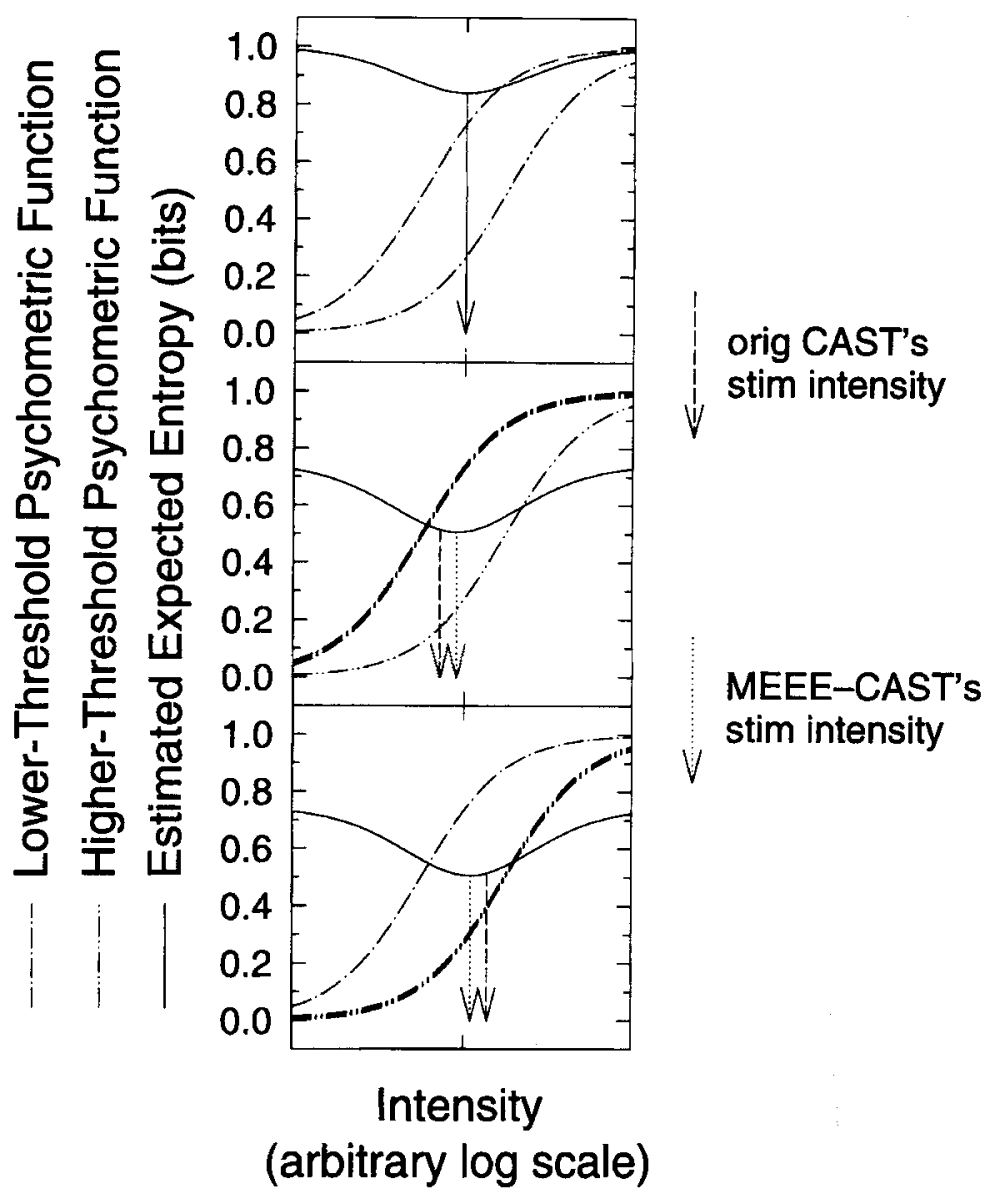

Figure 2. Illustration of how original CAST can select suboptimal stimuli. Top panel: The two psychometric functions are weighted equally, and original CAST and MEEE-CAST both test midway between the two thresholds. Middle panel: The lower threshold psychometric function (heavy broken line) is weighted heavily (0.787), and original CAST tests at too low an intensity. Bottom panel: The higher threshold psychometric function (heavy broken line) is weighted heavily (0.787), and original CAST tests at too high an intensity. CAST, classification of audiograms by sequential testing; MEEE, minimum estimated expected entropy. 
the two thresholds, as indicated by the dashed arrowheaded lines. Note that the specific values generated in these examples may be unrealistic, but their qualitative characteristics give an intuitive feel for MEEE-CAST and original CAST.

Equal weights. First consider the top panel of Figure 2, which represents the situation in which the current probability of each category is .5. In this toy example, the entropy of the current distribution across categories is 1 bit. Testing at extremely high intensities would be uninformative because either psychometric function would generate a correct response. Testing at extremely low intensities would also be uninformative because neither psychometric function would generate a correct response. This is why the estimated expected entropy for the next trial asymptotes at 1 bit for both very high and very low intensities: No information would be extracted, so the entropy after such a trial would remain at 1 bit.

In the top panel of Figure 2, both MEEE-CAST and original CAST select a stimulus intensity midway between the thresholds of the two categories, maximizing the information likely to be extracted from the trial. Lines representing both stimuli are therefore superimposed, represented by the solid arrowheaded line.

Lower threshold more likely. Next, consider the middle panel of Figure 2. Here, the lower threshold psychometric function is darker, representing the situation in which the current probability of the lower threshold category is high (.787 in this example). In this situation, the current entropy of the distribution across categories is $\sim 0.75$ bits, which is why the estimated expected entropy after the next trial asymptotes at $\sim 0.75$ bits for both very high and very low intensities.

In the middle panel of Figure 2, original CAST tests at a much lower intensity than does MEEE-CAST: MEEE-CAST and original CAST both move the stimulus intensity toward the more highly weighted threshold, but original CAST moves the stimulus intensity too far in this direction. If we assume that the correct threshold is the weighted mean of the thresholds of the two categories, MEEE-CAST's choice of stimulus intensity is a much better approximation of the best stimulus intensity than is original CAST's choice. (The best choice, which is not shown in Figure 2 because it would be virtually superimposed on MEEE-CAST's choice, is found by minimizing the actual expected entropy, which is found by substituting the true psychometric function, $\Psi(s)$-see Equation 13-for the estimated psychometric function, $\bar{\psi}^{(t-1)}(s)$, in the definition of estimated expected entropy found in Equation 9.)

Higher threshold more likely. Finally, consider the bottom panel of Figure 2 . Here, the higher threshold psychometric function is darker, representing the situation in which the current probabilities favor the higher threshold category (again, probability of .787, which again causes the estimated expected entropy after the next trial to asymptote at $\sim 0.75$ bits). This panel complements the middle panel: Whereas original CAST tests at too low an intensity in the middle panel, it tests at too high an intensity in the bottom panel.

When the lower threshold category provides a good approximation of the true psychometric function (middle panel of Figure 2), why is the optimal stimulus intensity higher than original CAST's choice? When the lower threshold category is highly weighted and its threshold coincides with the subject's true threshold, original CAST will test very close to the subject's true threshold. If the subject succeeds in response to such a stimulus, much information is extracted from the trial because it is highly unlikely that the subject would succeed in response to a low-intensity stimulus if the true threshold were high. However, when being tested at true threshold, the subject succeeds only $50 \%$ of the time. (This assumes a perfectly attentive subject; success is even less likely when subjects are inattentive.) Thus, on at least half of such trials, the subject fails, and very little information is extracted because higher threshold categories would also be consistent with such a failure. It is true that by increasing stimulus intensity away from true threshold, less information would be extracted when the subject succeeds because higher threshold categories would not be quite as inconsistent with such an outcome. However, this slight reduction in the information extracted by success trials is more than compensated for by the reduced probability of a failure trial, which would not be very informative. Of course, at some point, increasing stimulus intensity yields diminishing returns; but, by design, MEEE-CAST attempts to test at an intensity at which these two competing effects are optimally balanced. Although variance as a measure of efficiency is inapplicable to the problem of subject classification, the MEEE-CAST strategy in this case is nevertheless reminiscent of the strategy of optimizing the sweat factor (Green, 1990; Laming \& Marsh, 1988; Taylor, 1971; Taylor \& Creelman, 1967).

\section{Monte Carlo Simulation}

To evaluate the performance of the MEEE algorithm, the performance of MEEE-CAST was compared with that of original CAST via computer simulation.

Design. PTO $(\Pi)$, false-alarm rate $(\Phi)$, and CAST method (original vs. MEEE) were manipulated in a crossed fashion $(3 \times 3 \times 2$ design). $\Pi$ was set at 1.0 (completely attentive), 0.85 (moderately inattentive), or 0.7 (very inattentive), and $\Phi$ was set at $0.0,0.15$, or 0.3 . Pseudosubjects were run whose true audiograms matched each of the nine prototypes (categories) in Figure 1. One hundred pseudosubjects were run for each $\Pi \times \Phi \times$ CAST method $\times$ category combination. Pseudosubjects were run for 50 trials each, for a grand total of 810,000 simulated trials.

The numerical experiment had two dependent measures: specificity and sensitivity. Specificity was defined as the probability of correctly categorizing a normally hearing pseudosubject, that is, one whose true audiogram matched Prototype 1. (Pseudosubjects were regarded as correctly categorized if the a posteriori probability of 
the correct prototype was the highest among all the a posteriori probabilities of the prototypes.) Sensitivity was defined as the probability of correctly categorizing a hearing-impaired pseudosubject. The sensitivity measure pooled data across pseudosubjects whose true audiograms matched Prototypes 2-9, weighting the values by each category's a priori probability (i.e., by the $p_{c}^{(0)} \mathrm{s}$ ). Correct categorization of a hearing-impaired pseudosubject demanded not only identifying it as hearing impaired, but also correctly identifying the type of hearing impairment (i.e., identifying the correct prototype). (Note that sensitivity and specificity must identify a "normal" category. Although this is appropriate for an application such as CAST, it is not a general requirement of the MEEE method, since entropy is a symmetrical measure that quantifies the amount of information in the probability distribution across all categories.)

Results. Figure 3 presents the results of the numerical experiment. Each row presents data for a different value of true PTO ( $\Pi$ ), and each column presents data for a different value of true false-alarm rate $(\Phi)$. (Recall that the estimated PTO was held constant at $\varpi=.85$, and the estimated false-alarm rate was held constant at $\varphi=0.15$.) For each $\Pi \times \Phi$ combination, the upper graph plots specificity as a function of trial number, and the lower graph plots sensitivity as a function of trial number.

For both CAST methods, sensitivity approached $100 \%$ asymptotically. Accordingly, Figure 3 plots sensitivity on

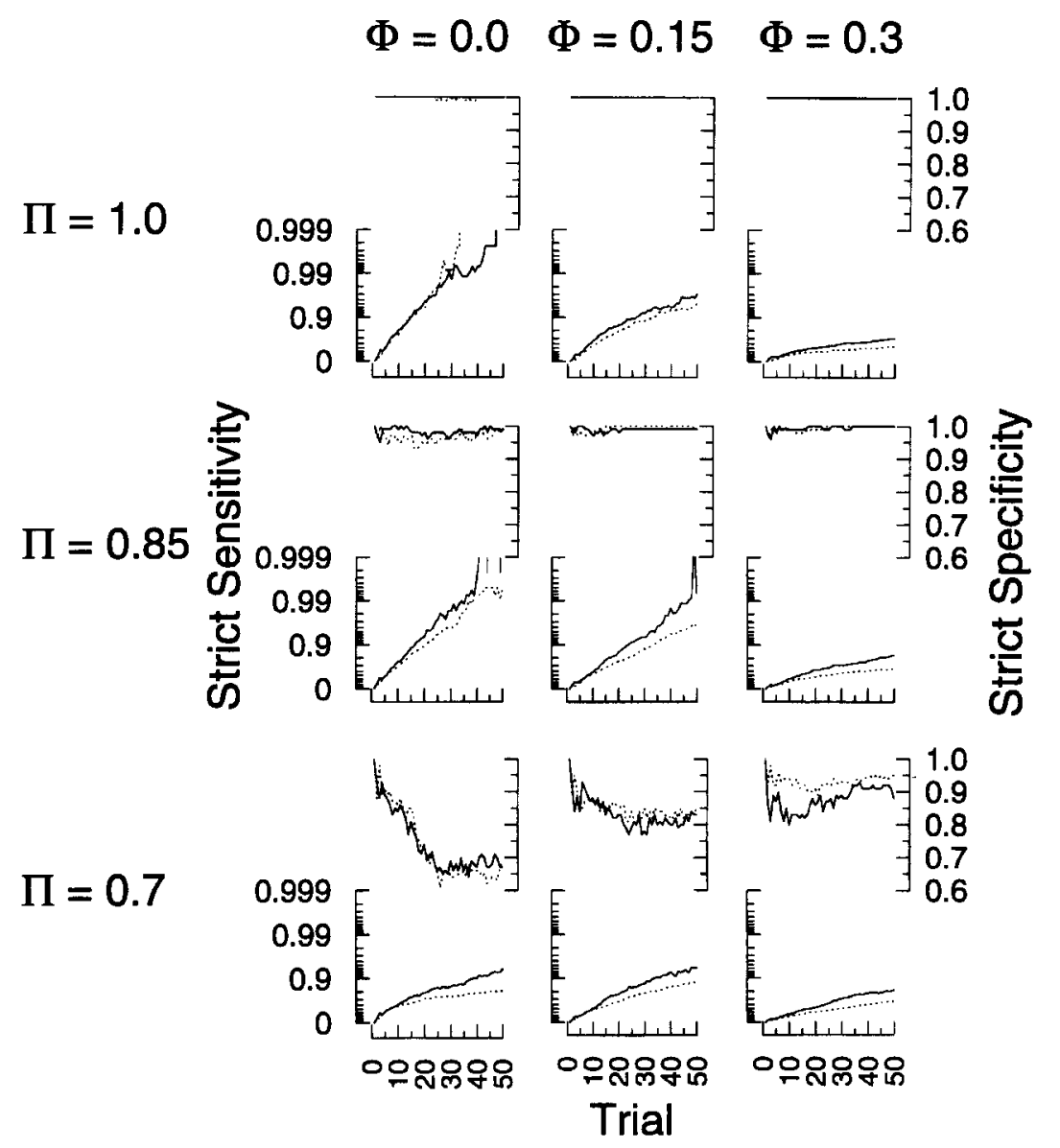

\section{MEEE-CAST orig CAST}

Figure 3. Sensitivity and specificity as a function of trial number for strict evaluation of CAST performance. Each of the three rows presents results for a different value of $I$, the true probability of task orientation (PTO), and each of three columns presents results for a different value of $\Phi$, the true false-alarm rate. Estimated PTO was held constant at $\varpi=0.85$, and estimated false-alarm rate was held constant at $\varphi=0.15$. Sensitivity, plotted on inverted logarithmic scales, refers to the probability of correctly categorizing a hearing-impaired pseudosubject (including the type of hearing impairment). Specificity, plotted on linear scales, refers to the probability of correctly categorizing a normally hearing pseudosubject. CAST, classification of audiograms by sequential testing; MEEE, minimum estimated expected entropy. 
inverted logarithmic scales. A positively sloped straight line on such a scale represents an exponential approach to sensitivity of $100 \%$. For nearly every $\Pi \times \Phi$ method combination, such straight lines on these scales accounted for the sensitivity data very well ( $r^{2}$ ranged from .817 to .993 , with the exception of original CAST for $\Pi=0.7$ and $\Phi=0.0$, where $r^{2}=.479$, indicating that the fit of the exponential approach was somewhat poorer). Because sensitivity started out at $0 \%$ for every $\Pi \times$ method combination, these fits were constrained to pass through $0 \%$ sensitivity at Trial 1 . Thus, the sole parameter in these fits was the rate of exponential approach, or, equivalently, the number of trials required to halve the difference between the current sensitivity and $100 \%$. This might be referred to as a method's "half-life," where short halflives indicate rapid approach to $100 \%$ sensitivity, and long half-lives indicate slow approach to $100 \%$ sensitivity. The best half-lives in Figure 3 occurred for $\Pi=1.0$ and $\Phi=0.0$, where original CAST had a half-life of 4.2 trials and MEEE-CAST had a half-life of 4.8 trials. The worst half-lives in Figure 3 occurred for $\Pi=1.0$ and $\Phi=0.3$, where original CAST had a half-life of 35.2 trials and MEEE-CAST had a half-life of 23.9 trials. In eight out of nine conditions, the half-life of MEEE-CAST was shorter than that of original CAST (i.e., the sensitivity of MEEE-CAST approached $100 \%$ faster than that of original CAST).

Although the sensitivity approached $100 \%$ exponentially in all conditions, its rate of approach was very slow for $\Phi=0.3$, so that after 50 trials (not an inconsequential number for testing an infant), the sensitivity of MEEECAST was only $70 \%-83 \%$, and the sensitivity of original CAST was only $56 \%-66 \%$. The reason for this poor performance is that CAST mistakes many of these subjects' false alarms for responses to the stimulus, and thus credits the subject with hearing a stimulus that really went unheard. This is an inherent problem for very high falsealarm rates and is precisely why clinical procedures for CAST and other forms of VRA include the insertion of "catch" trials on which no stimulus is presented, with the demand that testing be aborted if there are too many responses to these no-stimulus trials. Furthermore, testers are instructed to ensure that an infant is attentive but with head clearly unturned before initiating a trial, thereby minimizing the chance of false alarms (Eilers, personal communication, May 1, 1996). Because experienced testers are typically better at such judgments, their subjects tend to generate fewer false alarms than those of novice testers (Steffens, personal communication, April 11, 1996). In any event, because an infant can tolerate only relatively few trials, sensitivity for a false-alarm rate as high as $\boldsymbol{\Phi}=0.3$ is rendered unacceptably low for a clinical test, which tends to validate the requirement that the test be aborted if there are too many false alarms.

Specificity, plotted on linear axes, behaved differently. It was about equally high between the two CAST methods, although it fell as $\Pi$ fell. Specificity attained $100 \%$ for $\Pi=1.0$ (perfectly attentive pseudosubject) and re- mained close to $100 \%$ for $\Pi=0.85$ (moderately inattentive pseudosubject). For $\Pi=0.7$ (very inattentive pseudosubject), however, specificity fell dramatically with falling $\Phi$, so that it reached about $90 \%$ for $\Phi=0.3$, but fell to about $70 \%$ for $\Phi=0$. This represents the fact that when task orientation is low, CAST mistakes a normal subject's slight paucity of correct responses as reflecting an inability to hear stimuli. However, inspection of the data revealed that CAST tended to misclassify these normal subjects as having merely the mildest impairment (Audiogram Prototype 2). If the sensitivity and specificity criteria are relaxed by treating cross-classifications between the normal and mildly impaired categories (Audiogram Prototypes 1 and 2) as inconsequential, then specificity for $\Pi=0.7$ improves to acceptable levels. This is illustrated in Figure 4, which plots sensitivity and specificity under these lax criteria (as opposed to the strict criteria of Figure 3). Also note that lax sensitivity was somewhat better than strict sensitivity across the board, and that the lax sensitivity of MEEE-CAST approached $100 \%$ more rapidly than did the lax sensitivity of original CAST in nine out of nine conditions. (Halflives in Figure 4 were best for $\Pi=1.0$ and $\Phi=0.0$, where original CAST had a half-life of 2.5 trials and MEEE-CAST had a half-life of 1.9 trials; half-lives in Figure 4 were worst for $\Pi=1.0$ and $\Phi=0.3$, where original CAST had a half-life of 27.3 trials and MEEE-CAST had a half-life of 17.3 trials.) The fact that lax specificity was so much better than strict specificity for $\Pi=0.7$ suggests a modification to the clinical use of CAST: If an infant is classified with mild impairment, the tester should initiate some probe trials above a mildly impaired threshold (Audiogram Prototype 2) but below a deeply impaired threshold. If the infant fails to respond on some of these trials, the classification as mildly impaired versus normal should be deemed unreliable.

\section{DISCUSSION}

Bayesian categorization has often been applied to automated medical diagnosis (e.g., de Dombal, Leaper, Staniland, McCann, \& Horracks, 1972; Jilly, 1988; Wiener, Gabbai, \& Jaffe, 1987; Willcox, Lapage, Bascomb, \& Curtis, 1973). When coupled with an extensive database, it has been reported to classify disorders with an accuracy exceeding that of a physician (de Dombal, 1975). The MEEE method, described in the present paper, is directly applicable to a potentially broad range of such categorization tasks, in which one wishes to collect data that bear on a subject's category as efficiently as possible. Although one must surely acknowledge that it is unclear how broadly results like those of de Dombal et al. generalize, it is also true that the clinical utility of CAST, which applies a dynamic Bayesian scheme for the purpose of audiogram categorization, has been confirmed by clinical trial (Eilers et al., 1993). Consequently, CAST was modified to serve as a sample application of the MEEE method. The MEEE method provides a principled way to 

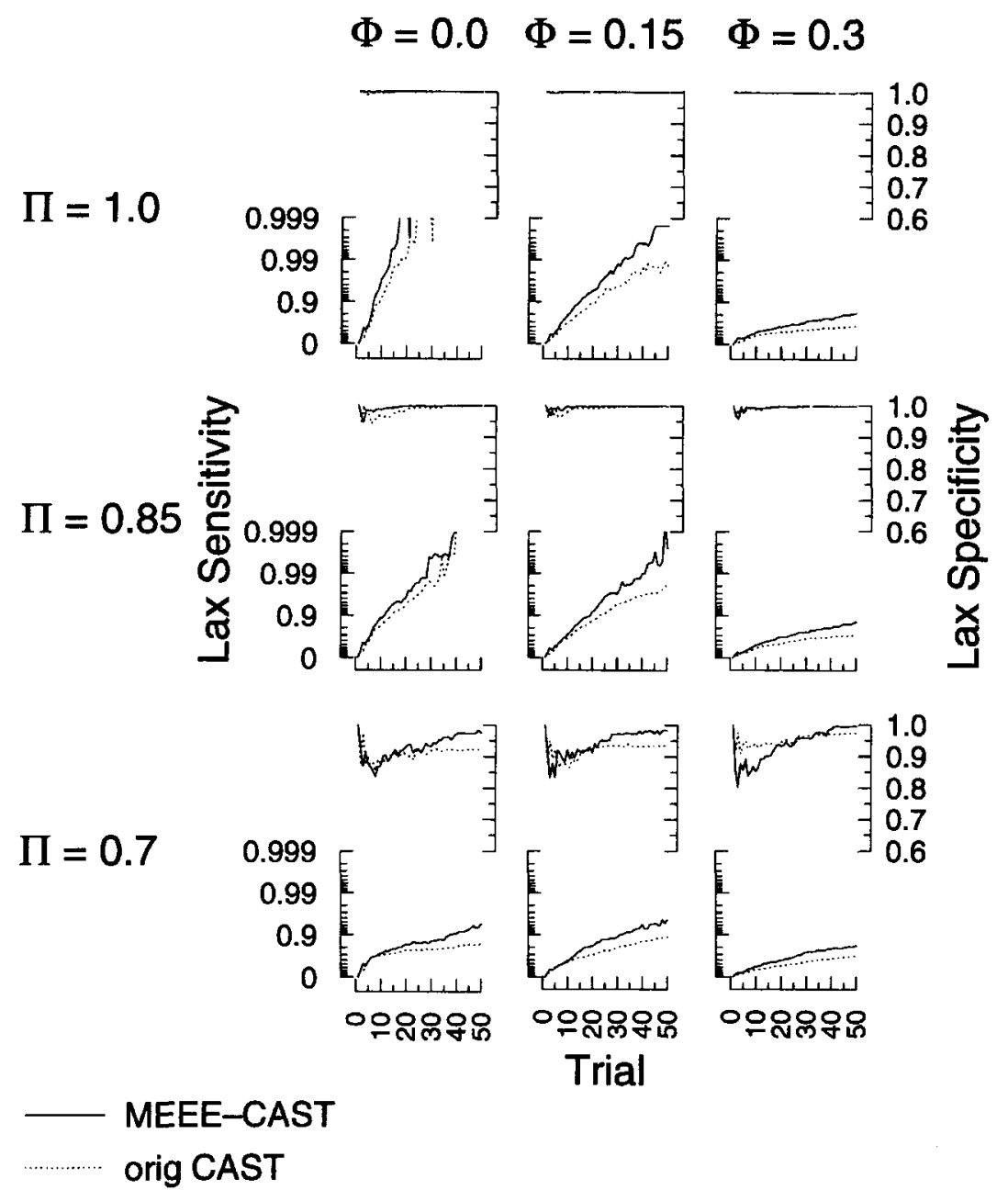

Figure 4. Sensitivity and specificity as a function of trial number for lax evaluation of CAST performance, whereby cross-classifications between normal hearing and mildest impairment (Categories 1 and 2) are regarded as inconsequential. For further details, see Figure 3 caption. CAST, classification of audiograms by sequential testing; MEEE, minimum estimated expected entropy.

select stimulus parameters for a broad range of procedures. In CAST, the stimulus parameters are acoustic frequency and intensity, but the MEEE method is also applicable to evaluation of visual characteristics, such as subject classification based on measurement of the spatial extent of the visual field (automated perimetry) or measurement of the contrast sensitivity function. For automated perimetry, the stimulus parameters would be vertical retinal position, horizontal retinal position, and luminance. For spatiotemporal contrast sensitivity, the stimulus parameters would be luminance contrast, spatial frequency, and temporal frequency. In all cases, by presenting stimuli explicitly designed to drive the entropy of the probability distribution across subject categories ever lower, the MEEE method attempts to extract the maximum amount of information from each trial so that tests can be terminated sooner without sacrificing reliability.

\section{Stopping Criterion}

The Monte Carlo simulations used to compare MEEECAST and original CAST followed each method through 50 trials. No tests were terminated before this point. (This is because of the interpretational difficulties involved in comparing a run that might terminate early with good reliability with another run that might terminate slightly later but with even better reliability.) In contrast, in a real laboratory or clinical situation, it might be desirable to complete the test at the point at which satisfactory reliability has been achieved. This will occur sooner for some runs than for others. A stopping criterion is therefore required. The natural stopping criterion for the MEEE method is to stop when the entropy is low enough. But how low is low enough? Original CAST stops testing when the probability of the most likely category meets or exceeds .9. An entropy at or below $\sim 0.469$ bits guarantees that the most likely category has 
a probability of at least .9 . In general (and stated here without proof), it is guaranteed that exactly one category has probability $\geq p$ (where we assume $p \geq .5$ ) when the entropy is $\leq-p \log p-(1-p) \log (1-p)$.

\section{Continuous Stimulus Parameters}

Original CAST treats its two stimulus parameters differently. It treats intensity as a continuous parameter by selecting stimulus intensity from a continuum of possible values, but it treats frequency as a discrete parameter, limited in the present case to $0.5,1,2$, or $4 \mathrm{kHz}$. Unlike original CAST, MEEE-CAST generalizes to an arbitrary number of stimulus parameters, even if some or all of these parameters are continuous rather than discrete, because it is formulated in terms of an abstract $N$-dimensional stimulus parameter, $s$. Because frequency, which in current and previous CAST work has been regarded as a discrete parameter, is really a continuous parameter, it would probably be beneficial to treat it as such. MEEE-CAST could then optimize the estimated expected entropy, $\tilde{H}(f, I)$, over the whole $(f, I)$ surface, rather than restricting attention to one-dimensional (constant- $I$ ) slices through the surface. Analogous approaches would also be applicable to automated perimetry and classification of spatiotemporal contrast sensitivity. These approaches would require defining prototypical thresholds across a continuous parameter, which, in turn, would require interpolation.

\section{Direct Categorization}

The problem solved by the particular implementation (CAST) chosen to illustrate the MEEE method could be approached instead as estimation of continuous parameters (e.g., via the minimum variance method, KingSmith, 1984, or the ideal psychometric procedure, Pelli, 1987), followed by categorization. If categorization is merely a secondary goal of the psychophysical procedure, such an approach might be preferred. However, if categorization is the primary goal (e.g., as in a screening program for hearing), then MEEE-CAST ought to accomplish the categorization more efficiently because it is designed specifically to maximize categorization efficiency. The MEEE method is also more obviously applicable to other categorization problems, such as distinguishing subjects with qualitatively different underlying characteristics (e.g., glaucoma or optic neuropathies, whose signs might be particularly obvious in automated perimetry; or M-system dysfunction of the visual system, whose signs might be particularly obvious in evaluation of spatiotemporal contrast sensitivity function).

The use of Bayesian statistics to dynamically update a posteriori probabilities has auspicious precedent in psychophysics (Green, 1993; Hall, 1968; Pentland, 1980; Watson \& Pelli, 1983). However, the goal of these antecedents is to maximize the reliability of a threshold estimate that is the same in kind as the stimulus parameter subject to manipulation. Other techniques relax this restriction (King-Smith, 1984; Pelli, 1987). However, even they are applicable only when estimating the value of a continuous parameter. In contrast, the method of minimum estimated expected entropy, in addition to being applicable to estimating the value of a continuous parameter, is also directly applicable to problems of categorization, in which a variable constructed to represent subject properties might possess only properties of a nominal scale (Stevens, 1951).

\section{REFERENCES}

CoRnsweEt, T. N. (1962). The staircase method in psychophysics. American Journal of Psychology, 75, 485-491.

DE Dombal, F. T. (1975). Computer assisted diagnosis of abdominal pain. In J. Rose \& J. Mitchell (Eds.), Advances in medical computing (pp. 10-19). New York: Churchill-Livingston.

de Dombal, F. T., Leaper, D. J., Staniland, J. R., McCanN, A. P., \& HorraCKS, J. S. (1972). Computer aided diagnosis of acute abdominal pain. British Medical Journal, 2, 9-13.

Eilers, R. E., Miskiel, E., Widen, J. E., \& Lopez, C. (1989, November). Audiometric testing in infancy: Computer models of infant responding. Paper presented at the American Speech-Language-Hearing Association Convention, St. Louis.

EILERS, R. E., ÖZdamar, Ö., \& STEFFENS, M. L. (1993). Classification of audiograms by sequential testing: Reliability and validity of an automated behavioral hearing screening algorithm. Journal of the American Academy of Audiology, 4, 172-181.

FindLAY, J. M. (1978). Estimates on probability functions: A more virulent PEST. Perception \& Psychophysics, 23, 181-185.

GREEN, D. M. (1990). Stimulus selection in adaptive psychophysical procedures. Journal of the Acoustical Society of America, 87, 2662-2674

GreEN, D. M. (1993). A maximum-likelihood method for estimating thresholds in a yes-no task. Journal of the Acoustical Society of America, 93, 2096-2105.

Gu, X., \& GREEN, D. M. (1994). Further studies of a maximum-likelihood yes-no procedure. Journal of the Acoustical Society of America, 96, 93-101.

HaLL, J. L. (1968). Maximum-likelihood sequential procedure for estimation of psychometric functions. Journal of the Acoustical Society of America, 44, 370.

HALL, J. L. (1981). Hybrid adaptive procedure for estimation of psychometric functions. Journal of the Acoustical Society of America, 69, $1763-1769$.

JILLY, B. J. (1988). Microcomputer application of Bayesian probability testing for the identification of bacteria. International Journal of BioMedical Computing, 22, 107-119.

Johnson, C. A., \& Shapiro, L. R. (1989). A comparison of MOBS (Modified Binary Search) and standard staircase test procedures in automated perimetry. In Noninvasive assessment of the visual system (1989 Technical Digest Series, Vol. 7, pp. 84-87). Washington, DC: Optical Society of America.

KING-SMITH, P. E. (1984). Efficient threshold estimates from yes-no procedures using few (about 10) trials. American Journal of Optometry \& Physiological Optics, 61, 119P.

King-Smith, P. E., Grigsby, S. S., Vingrys, A. J., Benes, S. C., \& SUPOWIT, A. (1994). Efficient and unbiased modifications of the QUEST threshold method: Theory, simulations, experimental evaluation and practical implementation. Vision Research, 34, 885-912.

LAMING, D., \& MARSH, D. (1988). Some performance tests of QUEST on measurements of vibrotactile thresholds. Perception \& Psychophysics, 44, 99-107.

LEVITT, H. (1971). Transformed up-down methods in psychoacoustics. Journal of the Acoustical Society of America, 49, 467-477.

Liden, G., \& KANKKUNEN, A. (1969). Visual reinforcement audiometry. Acta Otolaryngologica, 67, 281-292.

MOORE, J. M., WiLson, W. R., \& Thompson, G. (1977). Visual reinforcement of head-turn responses in infants under 12 months of age. Journal of Speech \& Hearing Disorders, 42, 328-334.

Özdamar, Ö, EIlers, R. E., Miskiel, E., \& Widen, J. (1990). Classifi- 
cation of audiograms by sequential testing using a dynamic Bayesian procedure. Journal of the Acoustical Society of America, 88, 21712179.

PELLI, D. G. (1987). The ideal psychometric procedure. Investigative Ophthalmology \& Visual Science, 28(Suppl.), 366.

Pentland, A. (1980). Maximum likelihood estimation: The best PEST. Perception \& Psychophysics, 28, 377-379.

Press, W. H., Teukolsky, S. A., Vetterling, W. T., \& Flannery, B. P. (1992). Numerical recipes in $C$ : The art of scientific computing (2nd ed.). New York: Cambridge University Press.

RoBBins, H., \& MonRo, S. (1951). A stochastic approximation method. Annals of Mathematical Statistics, 22, 400-407.

STEVENS, S. S. (1951). Mathematics, measurement, and psychophysics. In S. S. Stevens (Ed.), Handbook of experimental psychology (pp. 1-49). New York: Wiley.

SuzukI, T., \& OGIBA, Y. (1961). Conditioned orientation reflex audiometry. Archives of Otolaryngology, 74, 192-198.

TAYLOR, M. M. (1971). On the efficiency of psychophysical measurement. Journal of the Acoustical Society of America, 49, 505-508

TAYLOR, M. M., \& CREELMAN, C. D. (1967). PEST: Efficient estimates on probability functions. Journal of the Acoustical Society of America, 41, 782-787.

TREUTWEIN, B. (1995). Adaptive psychophysical procedures. Vision Research, 35, 2503-2522.

TYRRELl, R. A., \& OWENS, D. A. (1988). A rapid technique to assess the resting states of the eyes and other threshold phenomena: The Modified Binary Search (MOBS). Behavior Research Methods, Instruments, \& Computers, 20, 137-141.

Watson, A. B., \& Fitzhugh, A. (1990). The method of constant stimuli is inefficient. Perception \& Psychophysics, 47, 87-91.

Watson, A. B., \& Pelli, D. G. (1983). QUEST: A Bayesian adaptive psychometric method. Perception \& Psychophysics, 33, 113-120.

WATT, R. J., \& ANDREWS, D. P. (1981). APE: Adaptive probit estimation of psychometric functions. Current Psychological Reviews, 1, 205-214.

WeTHERILL, G. B., \& LEVITT, H. (1965). Sequential estimation of points on a psychometric function. British Journal of Mathematical \& Statistical Psychology, 18, 1-10.

Wiener, F., GabBai, M., \& JAFFE, M. (1987). Computerized classification of congenital malformations using modified Bayesian approach. Computers in Biology \& Medicine, 17, 259-267.

Willcox, W. R., Lapage, S. P., Bascomb, S., \& Curtis, M. A. (1973). Identification of bacteria by computer: Theory and programming. Journal of General Microbiology, 77, 317-330.

\section{APPENDIX A}

In order to simplify the dynamic update of the a posteriori distribution of category probabilities, we wish to derive the recurrence relation of Equation 3 from the familiar Bayesian formulation of Equation 2, which is

$$
p_{c}^{(t)}=\frac{p_{c}^{(0)} \prod_{\tau=1}^{t} \operatorname{Pr}\left(\mathbf{R}_{\tau}=R_{\tau} \mid \mathbf{C}=c\right)}{\sum_{\gamma}\left[p_{\gamma}^{(0)} \prod_{\tau=1}^{t} \operatorname{Pr}\left(\mathbf{R}_{\tau}=R_{\tau} \mid \mathbf{C}=\gamma\right)\right]},
$$

where $p_{c}^{(0)}$ represents the a priori probability of category $c$, and $\operatorname{Pr}\left(\mathbf{R}_{\tau}=R_{\tau} \mid \mathbf{C}=c\right)$ represents the conditional probability of the obtained response on trial $\tau$, given that $c$ is the correct category. This can be more succinctly expressed as

$$
p_{c}^{(t)}=\frac{n_{c}^{(t)}}{\sum_{\gamma} n_{\gamma}^{(t)}}
$$

where

$$
n_{c}^{(t)}=p_{c}^{(0)} \prod_{\tau=1}^{t} \operatorname{Pr}\left(\mathbf{R}_{\tau}=R_{\tau} \mid \mathbf{C}=c\right)
$$

is the numerator for category $c$ after trial $t$. By extracting the $t$ term from the product, one can rewrite Equation A3 as

$$
n_{c}^{(t)}=p_{c}^{(0)} \operatorname{Pr}\left(\mathbf{R}_{t}=R_{t} \mid \mathbf{C}=c\right) \prod_{\tau=1}^{t-1} \operatorname{Pr}\left(\mathbf{R}_{\tau}=R_{\tau} \mid \mathbf{C}=c\right) .
$$

But, by the definition in Equation A3,

$$
n_{c}^{(t-1)}=p_{c}^{(0)} \prod_{\tau=1}^{t-1} \operatorname{Pr}\left(\mathbf{R}_{\tau}=R_{\tau} \mid \mathbf{C}=c\right)
$$

Therefore, Equation A4 can be re-expressed as a recurrence relation:

$$
n_{c}^{(t)}=\operatorname{Pr}\left(\mathbf{R}_{t}=R_{t} \mid \mathbf{C}=c\right) n_{c}^{(t-1)} .
$$

Substituting this into Equation A2 yields

$$
p_{c}^{(t)}=\frac{\operatorname{Pr}\left(\mathbf{R}_{t}=R_{t} \mid \mathbf{C}=c\right) n_{c}^{(t-1)}}{\sum_{\gamma}\left[\operatorname{Pr}\left(\mathbf{R}_{t}=R_{t} \mid \mathbf{C}=\gamma\right) n_{\gamma}^{(t-1)}\right]} .
$$

But Equation A2 is an implicit definition that allows us to write $n_{c}^{(t-1)}$ as $p_{c}^{(t-1)} \sum_{\gamma^{\prime}} n_{\gamma^{\prime}}^{(t-1)}$ and that also allows us to write $n_{\gamma}^{(t-1)}$ as $p_{\gamma}^{(t-1)} \sum_{\gamma^{\prime}} n_{\gamma^{\prime}}^{(t-1)}$. Making these two substitutions in Equation A7 and then canceling the numerator's $\Sigma_{\gamma}, n_{\gamma^{\prime}}^{(t-1)}$ term with the same term in the denominator yields

$$
p_{c}^{(t)}=\frac{p_{c}^{(t-1)} \operatorname{Pr}\left(\mathbf{R}_{t}=R_{t} \mid \mathbf{C}=c\right)}{\sum_{\gamma}\left[p_{\gamma}^{(t-1)} \operatorname{Pr}\left(\mathbf{R}_{t}=R_{t} \mid \mathbf{C}=\gamma\right)\right]},
$$

which is Equation 3, as desired. The advantage of using this recurrence relation over using the more familiar standard Bayesian expression of Equations 2 and $\mathrm{A} 1$ is that the recurrence relation expresses the a posteriori probabilities after trial $t$ as a function solely of the probabilities after trial $t-1$ and of the response to trial $t$. The recurrence relation eliminates the need to compute several products with ever-increasing numbers of terms.

\section{APPENDIX B}

We wish to derive the expression of the expected entropy in Equation 9 from the definition of Equation 8 . The definition is

$$
\tilde{H}^{(t)}(s)=\bar{\psi}^{(t-1)}(s) \breve{H}^{(t)}(s)+\left[1-\bar{\psi}^{(t-1)}(s)\right] \vec{H}^{(t)}(s),
$$

where $\bar{\psi}^{(t-1)}(s)$, defined in Equation 7, is the mean of the psychometric functions, weighted by the a posteriori probabilities after trial $t-1$.

Consider one term of the definition - the conditional entropy, contingent on a success in response to stimulus $s$ :

$$
\breve{H}^{(t)}(s)=-\sum_{c} \breve{p}_{c}^{(t)}(s) \log \bar{p}_{c}^{(t)}(s)
$$

where $\breve{p}_{c}^{(I)}(s)$ is the a posteriori probability of category $c$, contingent on a success in response to stimulus $s$ on trial $t$. By the re- 
currence relation of Equations 3 and A8, the components of this conditional entropy are

$$
\tilde{p}_{c}^{(t)}(s)=\frac{p_{c}^{(t-1)} \psi_{c}(s)}{\sum_{\gamma}\left[p_{\gamma}^{(t-1)} \psi_{\gamma}(s)\right]}
$$

where $\psi_{c}(s)$ has been substituted in for $\operatorname{Pr}\left(\mathbf{R}_{t}=R_{t} \mid C=c\right)$ because we are interested in the probability contingent on a success, and the probability of a success under category $c$ is precisely what is given by the psychometric function $\psi_{c}(s)$. That is, $\psi_{c}^{(s)}=\operatorname{Pr}($ success $\mid \mathbf{C}=c)$. Now, note that the denominator in Equation B3 constitutes the definition of $\bar{\psi}^{(t-1)}(s)$. Equation B3 thus can be rewritten as

$$
\bar{p}_{c}^{(t)}(s)=\frac{p_{c}^{(t-1)} \psi_{c}(s)}{\bar{\psi}^{(t-1)}(s)} .
$$

Substituting this result into the definition of the conditional entropy contingent on a success from Equation B2 yields

$$
\check{H}^{(t)}(s)=-\sum_{c} \frac{p_{c}^{(t-1)} \psi_{c}(s)}{\bar{\psi}^{(t-1)}(s)} \log \frac{p_{c}^{(t-1)} \psi_{c}(s)}{\bar{\psi}^{(t-1)}(s)} .
$$

Now consider another term of the definition from Equation B1 - the conditional entropy contingent on a failure in response to stimulus $s$ :

$$
\bar{H}^{(t)}(s)=-\sum_{c} \hat{p}_{c}^{(t)}(s) \log \hat{p}_{c}^{(t)}(s) .
$$

By the recurrence relation of Equations 3 and A8, the components of this conditional entropy are

$$
\hat{p}_{c}^{(t)}(s)=\frac{p_{c}^{(t-1)}\left[1-\psi_{c}(s)\right]}{\sum_{\gamma}\left\{p_{\gamma}^{(t-1)}\left[1-\psi_{\gamma}(s)\right]\right\}},
$$

where $1-\psi_{c}(s)$ has been substituted in for $\operatorname{Pr}\left(\mathbf{R}_{t}=R_{t} \mid C=c\right)$ because we are interested in the probability contingent on failure. Distributing the summation in the denominator and again recognizing the definition of $\bar{\psi}^{(t-1)}(s)$ yields

$$
\hat{p}_{c}^{(t)}(s)=\frac{p_{c}^{(t-1)}\left[1-\psi_{c}(s)\right]}{1-\bar{\psi}^{(t-1)}(s)}
$$

Substituting this result into the definition of the conditional entropy contingent on failure in Equation B6 yields

$$
\hat{H}^{(t)}(s)=-\sum_{c} \frac{p_{c}^{(t-1)}\left[1-\psi_{c}(s)\right]}{1-\bar{\psi}^{(t-1)}(s)} \log \frac{p_{c}^{(t-1)}\left[1-\psi_{c}(s)\right]}{1-\bar{\psi}^{(t-1)}(s)} .
$$

Finally, substitute the conditional entropy expressions of Equations B5 and B9 into the definition of estimated expected entropy in Equation B1. Cancellation of the $\bar{\psi}^{(l-1)}(s)$ in the success term's denominator and the $1-\bar{\psi}^{(t-1)}(s)$ in the failure term's denominator yields

$$
\begin{aligned}
\tilde{H}^{(t)}(s)= & -\sum_{c} p_{c}^{(t-1)} \psi_{c}(s) \log \frac{p_{c}^{(t-1)} \psi_{c}(s)}{\bar{\psi}^{(t-1)}(s)} \\
& -\sum_{c} p_{c}^{(t-1)}\left[1-\psi_{c}(s)\right] \log \frac{p_{c}^{(t-1)}\left[1-\psi_{c}(s)\right]}{1-\bar{\psi}^{(t-1)}(s)} .
\end{aligned}
$$

Summing termwise, factoring out $p_{c}^{(t-1)}$, and using the identity $x \log y=\log y^{x}$ yields

$$
\begin{aligned}
& \tilde{H}^{(t)}(s)= \\
& \quad-\sum_{c} p_{c}^{(t-1)} \log \left[p_{c}^{(t-1)}\left(\frac{1-\psi_{c}(s)}{1-\bar{\psi}^{(t-1)}(s)}\right)^{1-\psi_{c}(s)}\left(\frac{\psi_{c}(s)}{\bar{\psi}^{(t-1)}(s)}\right)^{\psi_{c}(s)}\right],
\end{aligned}
$$

which is Equation 9, as desired. Thus, after calculating $\bar{\psi}^{(t-1)}(s)$ through a simple weighted sum, one can calculate the expected entropy for stimulus $s$ via a single relation.

\section{APPENDIX C}

The MEEE method requires that before every trial, the stimulus parameters, $s$, be selected so that the estimated expected entropy, $\tilde{H}$, is minimized. The appropriate numerical techniques for accomplishing this minimization may depend on the nature of the components of the $N$-dimensional stimulus vector, $s$, but standard techniques, such as Levenberg-Marquardt minimization (e.g., Press, Teukolsky, Vetterling, \& Flannery, 1992), will probably usually suffice. For the sample MEEE implementation of the present paper (MEEE-CAST), we have a two-dimensional vector of stimulus parameters, $s=(f, I)$. However, CAST treats frequency as a discrete parameter (taking on the values $0.5,1,2$, or $4 \mathrm{kHz}$, as illustrated in Figure 1). Thus, the twodimensional minimization problem may be reduced to four separate one-dimensional minimization problems, followed by selection of the best of the four minimizations. Figure $\mathrm{Cl}$, in which the solid line plots $\tilde{H}^{(1)}(f, I)$ for $f=500 \mathrm{~Hz}$, illustrates the first iteration for Trial 1 in such a one-dimensional minimization.

For each successive fixed $f$, the minimization was accomplished by a quadratic approximation. Although not as efficient as Levenberg-Marquardt minimization, it did converge much more rapidly than did gradient descent. The quadratic approximation assumes that the curve relating $\tilde{H}$ to $I$ behaves locally as a second order polynomial, that is, that

$$
\tilde{H}^{(t)}(f, I) \approx A I^{2}+B I+C,
$$

where $A, B$, and $C$ are unknown constants. The first derivative with respect to $I$ is therefore

$$
\frac{\partial \tilde{H}^{(t)}(f, I)}{\partial I} \approx 2 A I+B
$$

$\tilde{H}^{(t)}(f, I)$ is minimized when the derivative is zero, that is, where $I \approx-B /(2 A)$. Thus, the $i$ th guess for the intensity, $I_{i}$, that minimizes $\tilde{H}^{(t)}(f, I)$ will be immediately computable as soon as the constants $A$ and $B$ are known. (Note that the constant $C$ in Equation $\mathrm{Cl}$ is therefore irrelevant.) To find these constants, evaluate the first derivative for the two immediately preceding guesses, $I_{i-1}$ and $I_{i-2}$, generating two equations in two unknowns:

$$
\begin{gathered}
\dot{\tilde{H}}_{i-1}=\left.\frac{\partial \tilde{H}^{(t)}(f, I)}{\partial I}\right|_{I=I_{i-1}} \approx 2 A I_{i-1}+B \\
\dot{\tilde{H}}_{i-2}=\left.\frac{\partial \tilde{H}^{(t)}(f, I)}{\partial I}\right|_{l=I_{i-2}} \approx 2 A I_{i-2}+B
\end{gathered}
$$

(Note that another method of quadratic approximation might find second derivatives of Equation $\mathrm{Cl}$ and evaluate the expressions 


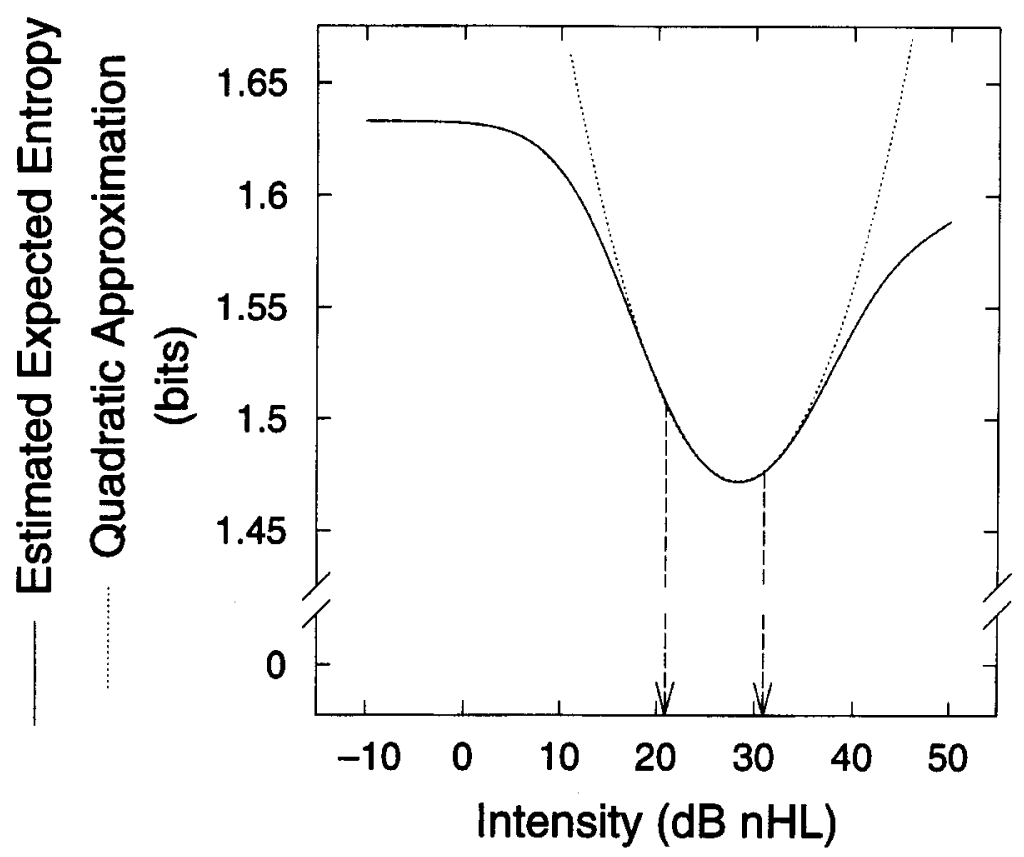

Figure C1. The first quadratic-approximation iteration in minimizing the estimated expected entropy of Trial 1 for stimulus frequency $(f=500 \mathrm{~Hz})$. Solid line plots $\tilde{H}^{(1)}(f, I)$ for $f=500 \mathrm{~Hz}$, and dotted line plots its quadratic approximation for $I_{-2} \approx 21 \mathrm{~dB} \mathrm{nHL}$ (left arrowheaded dashed line) and $I_{-1} \approx 31 \mathrm{~dB} \mathrm{nHL}$ (right arrowheaded dashed line).

at only one intensity. The current method instead takes only first derivatives of Equation $\mathrm{Cl}$ but evaluates the expressions at two intensities. One might analogize the distinction to solving a problem via a single second order differential equation versus solving it via two coupled first order differential equations.) Solving Equation $C 3$ for the two unknowns $A$ and $B$ (which implicitly generates the quadratic approximation depicted by the dotted line in Figure $\mathrm{C} 1$ ) and applying the aforementioned formula $I_{i}=-B /(2 \mathrm{~A})$ yields

$$
I_{i}=\frac{\dot{\tilde{H}}_{i-2} I_{i-1}-\dot{\tilde{H}}_{i-1} I_{i-2}}{\dot{\tilde{H}}_{i-2}-\dot{\tilde{H}}_{i-1}} .
$$

Thus, as long as the estimated expected entropy of Equation 9 is differentiable, Equation $\mathrm{C} 4$ gives the next guess as a function of the two preceding guesses. Indeed, Equation 9 is differentiable, yielding

$$
\begin{aligned}
\dot{H}^{(t)}(f, I) & =\frac{\partial \tilde{H}^{(t)}(f, I)}{\partial I} \\
& =\sum_{c} p_{c}^{(t-1)} \dot{\psi}_{c}(f, I) \ln \left[\frac{\bar{\psi}^{(t-1)}(f, I)}{1-\bar{\psi}^{(t-1)}(f, I)} \cdot \frac{1-\psi_{c}(f, I)}{\psi_{c}(f, I)}\right],
\end{aligned}
$$

where $\dot{\psi}_{c}(f, I)=\partial \psi_{c} / \partial I$, and, to minimize complications, the base of the logarithm is taken to be $e$, so that the entropy is measured in natural units (nats), easily convertible at procedure's end to bits via the relation $x$ nats $=(x / \ln 2)$ bits $\approx 1.44 x$ bits. Differentiation of the formula for $\psi_{c}(f, I)$ from Equation 12 yields

$$
\dot{\psi}_{c}(f, I)=\frac{k(\omega-\varphi)}{4 \cosh ^{2} \frac{k\left(s-T_{c, f}\right)}{2}},
$$

completing the statement in Equation $\mathrm{C} 5$ of the required derivative of the estimated expected entropy.

Finally, in order to start the process of iterating to a solution, we need to define the first two guesses, $I_{-2}$ and $I_{-1}$. One of these was taken as the preferred intensity of original CAST at the given frequency, that is,

$$
I_{-2}=\sum_{c} p_{c}^{(l-1)} T_{c, f}
$$

To choose the other guess, examine the slope $\dot{\tilde{H}}^{(t)}\left(f, I_{-2}\right)$. If the quadratic approximation is reasonable, a negative slope implies that $I_{-2}$ is to the left of the minimum, and a positive slope implies that $I_{-2}$ is to the right of the minimum (see Figure $\mathrm{Cl}$ ). Thus, if $\tilde{H}^{(t)}\left(f, I_{-2}\right)$ is negative, we can bracket the minimum between $I_{-2}$ and $I_{-1}$ by setting $I_{-1}=I_{-2}+(5 \mathrm{~dB}) \Delta$, where $\Delta$ is the smallest positive integer that yields a positive value for $\tilde{H}^{(t)}\left(f, I_{-1}\right)$. On the other hand, if $\tilde{H}^{(t)}\left(f, I_{-2}\right)$ is positive, we can bracket the minimum between $I_{-2}$ and $I_{-1}$ by setting $I_{-1}=I_{-2}-(5 \mathrm{~dB}) \Delta$, where $\Delta$ is now the smallest positive integer that yields a negative value for $\tilde{H}^{(t)}\left(f, I_{-1}\right)$. The dashed arrowheaded lines in Figure $\mathrm{C} 1$ represent $I_{-2} \approx 21 \mathrm{~dB} \mathrm{HL}$ and $I_{-1} \approx 31 \mathrm{~dB} \mathrm{HL}$, on which the quadratic approximation depicted by the dotted line in Figure $\mathrm{Cl}$ was based. Note that the solid and dotted lines have minima at very nearly the same intensity, illustrating how fast the quadratic approximation can converge.

Bracketing the minimum in such a manner guarantees that $I_{0}$ will lie between $I_{-2}$ and $I_{-1}$, and efficiently drives $I_{0}$ toward a min- 
imum. To increase efficiency, this principle was actually applied to all iterations of the minimization. Namely, instead of iteration $i$ being governed by Equation $\mathrm{C} 4$, it was governed by

$$
I_{i}=\frac{\dot{\tilde{H}}_{j} I_{i-1}-\dot{\tilde{H}}_{i-1} I_{j}}{\dot{\tilde{H}}_{j}-\dot{\tilde{H}}_{i-1}}
$$

where $j$ is the largest integer less than $i-1$ such that $\dot{\tilde{H}}_{j}$ has sign opposite of $\tilde{H}_{i-1}$. Note that if these procedures converge, they will converge at a local minimum rather than a local maximum. Indeed, in many attempts, including the 1.62 million required for the present paper's MEEE-CAST trials, these procedures never failed to converge.
The reader may note that this appendix finds a local minimum rather than a global minimum. However, if the guess for the best intensity in original CAST is relatively close to the actual best intensity, these methods ought to converge to the desired value. In any event, if these methods ever fail to find the best value, it would serve to render this paper's MEEE-CAST implementation suboptimal; thus MEEE-CAST ought to perform even better if the minimization method used in this appendix were replaced with a better one.

(Manuscript received December 27, 1995; revision accepted for publication September 21, 1996.) 